\title{
Performance Evaluation of Polygon-based and Voxel-based Finite Element Analysis on Tibia with Osteogenesis Imperfecta
}

\author{
H.Y. Tan ${ }^{1}$, K. S. Basaruddin*1 ${ }^{1}$, S. F. Khan ${ }^{1}$, M.H. Mat Som ${ }^{1}$, A. R. Sulaiman ${ }^{2}$ \\ ${ }^{1}$ Medical Devices Research Group, School of Mechatronic Engineering, Universiti Malaysia Perlis, Pauh Putra Campus, 02600, \\ Arau, Perlis, Malaysia. \\ ${ }^{2}$ Department of Orthopaedics, School of Medical Science, Universiti Sains Malaysia, 16150 Kubang Kerian, Kelantan, Malaysia. \\ khsalleh@unimap.edu.my
}

\begin{abstract}
Osteogenesis Imperfecta (OI) is a bone disease that commonly can cause bone deformation and could cause death in extreme cases. To treat this disease, rodding surgeries are required. Such surgeries require pre-surgery planning to evaluate the fracture risk. Finite element analysis (FEA) has been widely used as computational tool to predict bone fracture. It is known that the accuracy of FEA is strongly dependent on discretization of element. There are two common types of element that is being used for bone analysis, namely polygon-based and voxel-based. Current clinical practices are more inclined to creating voxel-based FE models based on patient specific computed tomography (CT) image as it is more accessible to them. Polygon-based FE models are less commonly used as medical personnel usually does not has the engineering expertise in handling FEA. However, there might be benefits in employing polygon-based models. This paper aims to compare the FEA performance between both types of FE models, and then suggest the better option for clinical usage. It was found that in most cases, the FEA predictions of both models were similar with percentage difference of $<1 \%$. However, polygon-based models showed its advantage when it comes to computing demand at it was able to perform FEA as much as 45 times faster than voxel-based model. To conclude, polygon-based FE model could be the better option for clinical uses for offering identical accuracy with less computing demand.
\end{abstract}

Key words : Finite element analysis, mesh, Osteogenesis Imperfecta, polygon, voxel

\section{INTRODUCTION}

Osteogenesis Imperfecta (OI), otherwise known as brittle bone disease, is a genetic bone fragility disorder caused by bone deformities, such as bowing of long bone. OI occurs fairly commonly among children with a statistical incidence of 1 in 10,000 to 1 in 20,000 newborns. OI is a heterogeneous disorder and is categorized into OI type I, type II, type III and type IV based on clinical, radiographic and genetic criteria [1]. Among these different types of OI, type II is regarded as one of the most lethal and regularly seen severe skeletal dysplasia, which may cause death [2].
Usually, rodding surgery will be required for OI patients. Doctors will predict the timing of fracture of bone in order to determine a suitable timing to perform the surgery. At present, the prediction of bone fracture is performed based on doctors' judgement with their experience. However, such approach is subjective in nature, therefore a quantitative method to determine the fracture risk is desired. Therefore, patient-specific prediction of bone fracture through finite element analysis (FEA) could enhance the surgery planning for OI patients. FEA is capable of performing static structural analysis with high reproducibility of the results [3], [4]. Such is the reason why there are finite element prediction of bone mechanical behaviours received attention from various researchers [5]-[9]. Besides, FEA has been proven to be able to predict mechanical behaviours of bone with relatively high accuracy [10]-[15].

Patient specific image of bone can be obtained through computed tomography (CT) or magnetic resonance imaging (MRI) scans, subsequently these images can be used to create finite element (FE) models. In principle, there are two types of FE models that can be derived from these imaging techniques, namely "polygon-based" and "voxel-based" FE models [16]. Polygon-based models are built based on polygon meshing that portrays the contours of bones. On the other hand, voxel-based models are geometric description of the bone built with multiple layers of three-dimensional volumetric pixels known as voxel. The FE models derived from CT scanning usually comes in the form of voxel-based models as such image is more easily attainable by medical personnel [17]. Such voxel-based models of human bones have high computational demands. This is due to the high resolution of the image data as a result of homogenous finite element generation in the overall microstructure [18].

During surgery planning, the prediction of fracture timing might involve multiple trials of FEA, this in turn will create more computational demand. Ideally, it would be better to keep computational demand low to save time and cost. As FEA is sensitive, small variations in parameters such as mesh type and geometry would affect the outcome of prediction [12], [19]. Therefore, this paper aims to investigate the influence of voxel-based and polygon-based 
finite element models in predicting the fracture of OIaffected tibia. The comparison between two types of mesh will provide additional insight on the reliability of simplified FE model namely polygon-based models, in order to reduce the computational demand. Furthermore, the difference between FE prediction on OI tibia using polygon-based and voxel-based mesh was investigated as well.

\section{METHODS}

Polygon-based and voxel-based FE models were created in this work. The models were compared in terms of FEA predictions under same boundary conditions to investigate the difference between the two FEA approaches. On top of that, the time taken for FEA to complete were recorded as well to evaluate the computing demand of both types of models.

\subsection{Development of OI tibia models}

A 3D geometrical model of a standard tibia was modified using ANSYS Workbench to artificially impose bowing of bone on the tibia. A set of $10 \mathrm{OI}$ tibia were created with varying bowing angles $\left(5^{\circ}, 7^{\circ}, 10^{\circ}, 12^{\circ}, 14^{\circ}, 15^{\circ}, 18^{\circ}\right.$, $20^{\circ}, 22^{\circ}, 25^{\circ}$, then these models were used in ANSYS Workbench to perform polygon-based FEA. Also, the models were exported in .STL format, subsequently these image files were used in Voxelcon (Quint Corp., Tokyo) software to create voxel-based models. Figure.1 shows the sample of polygon-based and voxel-based models.

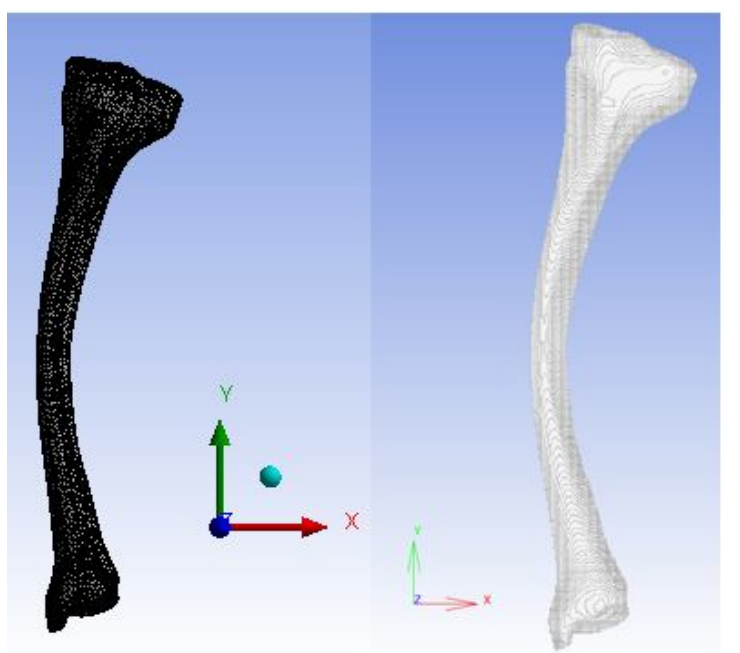

Figure 1: Polygon-based models (left) and voxel based models (right)

\subsection{Polygon-based FEA}

A set of 10 tibia models were used in ANSYS Workbench to simulate forces reacting on OI tibia during stance. Fine mesh was created for the models with a mesh size of $2 \mathrm{~mm}$. The loading on tibia during stance is derived based body weight and weighing factor, which in this case was set as $1.07 \mathrm{BW}$ [20]. A mass of $65 \mathrm{~kg}$ was selected throughout the FEA, which results in an applied load of $682.3 \mathrm{~N}$ during stance. For the boundary condition, load was applied on tibiofemoral joint, while fixed support was set at the ankle joint. Then, FEA was executed and maximum vonMises stress and the time taken for the analysis was recorded.

\subsection{Voxel-based FEA}

The same set of tibia models were exported to Voxelcon in .STL format to build voxel-based FE models. For the boundary conditions, voxels on the surface of tibiofemoral joint were selected to resemble the load applied on the polygon-based model. Similarly, fixed support was applied on the surface voxels at the knee joint, based on the boundary condition of polygon-based FE model. The same load of $682.3 \mathrm{~N}$ was applied for this FEA as well. Figure. 2 shows the regions of load applied of both polygon-based and voxel-based model, while Figure. 3 shows the regions selected for fixed support applied on these models.

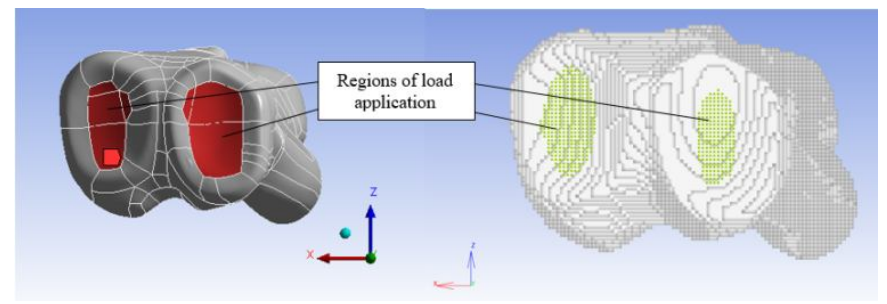

Figure 2: Regions of load application of polygon-based (left) and voxel-based (right) models

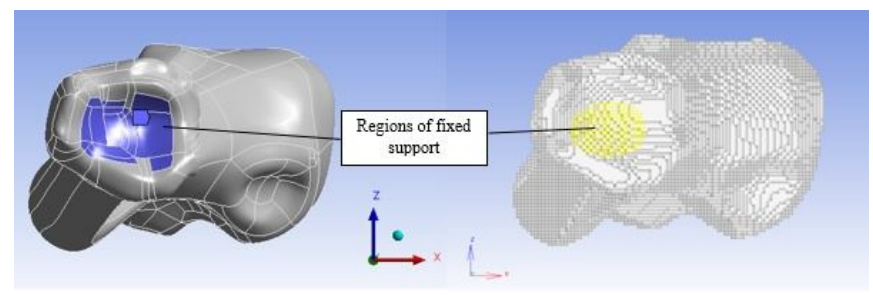

Figure 3: Regions of fixed support of polygon-based (left) and voxel-based (right) models

\subsection{Fracture load prediction of both models}

Both the polygon-based and voxel-based FEA models were used to compare the ability to predict fracture load of OI tibia. The models were tested to predict fracture due to compressive load and loads from sagittal plane and coronal plane direction. The tibia was assumed have fracture occur when von-Mises stress reaches $115 \mathrm{MPa}$ [21]. Therefore, artificial loads from various directions were imposed on the tibia until the fracture load is reached. The OI tibia model with bowing angle of $15^{\circ}$ was selected to carry out the fracture load prediction. Figure. 4 shows the illustration of the various load direction. 


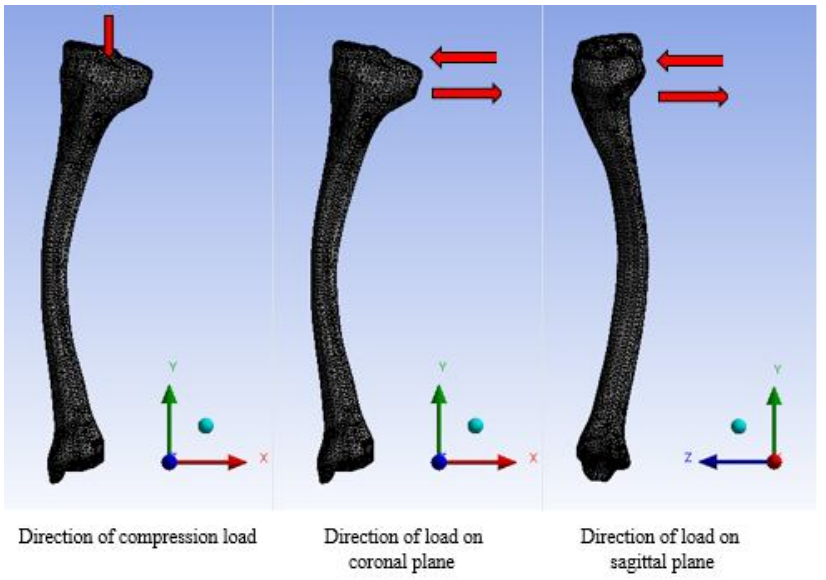

Figure 4: Various direction of loading

\section{RESULTS AND DISCUSSION}

\subsection{FEA prediction during stance}

The FEA results of both polygon-based and voxel-based models were compared in terms of maximum von-Mises stress. By comparing voxel-based FEA results to polygonbased results, it was observed that the percentage difference ranges from 0.029 to $7.33 \%$. On overall, the average percentage difference sits at $0.166 \%$, the results are shown in Figure. 5.

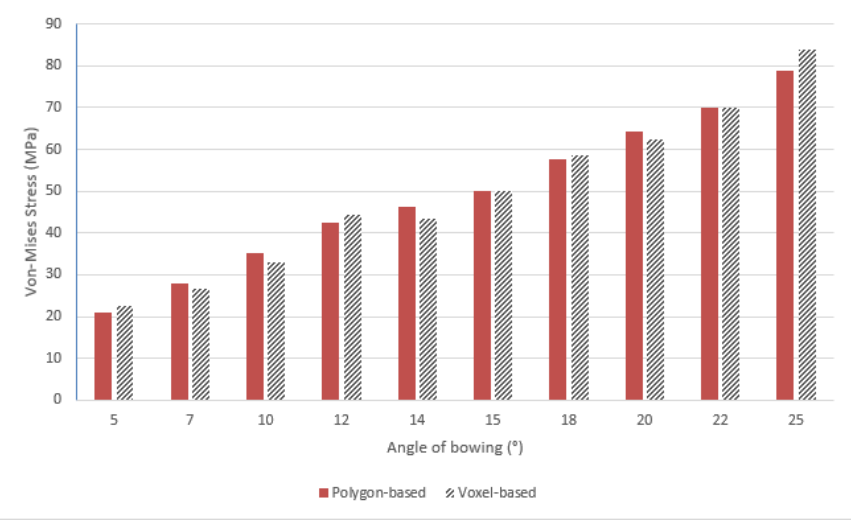

Figure 5: Plot of FEA results of polygon-based and voxel-based models

\subsection{Fracture load prediction}

For compressive stress fracture, the polygon-based model predicted fracture to occur when compressive load reaches $1568 \mathrm{~N}$, while voxel-based model predicted $1710 \mathrm{~N}$, this yields a percentage difference of $8.30 \%$. As for fracture caused by loading on sagittal plane direction, polygon-based model predicted the fracture load at $431 \mathrm{~N}$, while voxel-based model predicted $427 \mathrm{~N}$, the percentage difference between these values are $0.928 \%$. Furthermore, for fracture on coronal plane direction, the polygon-based model predicted the load to be $410 \mathrm{~N}$, while voxel-based model predicted $411 \mathrm{~N}$, and the percentage difference is $0.244 \%$.

\subsection{Critical stress regions}

Both of the models were also compared in terms of the prediction of stress distribution. The FEA results of compression load were taken to observe the critical stress region where the fracture would occur, the location of critical stress region of both models were predicted to be located at the middle of the bone and are identical. Figure 6 shows the stress distribution and critical stress region of both models.

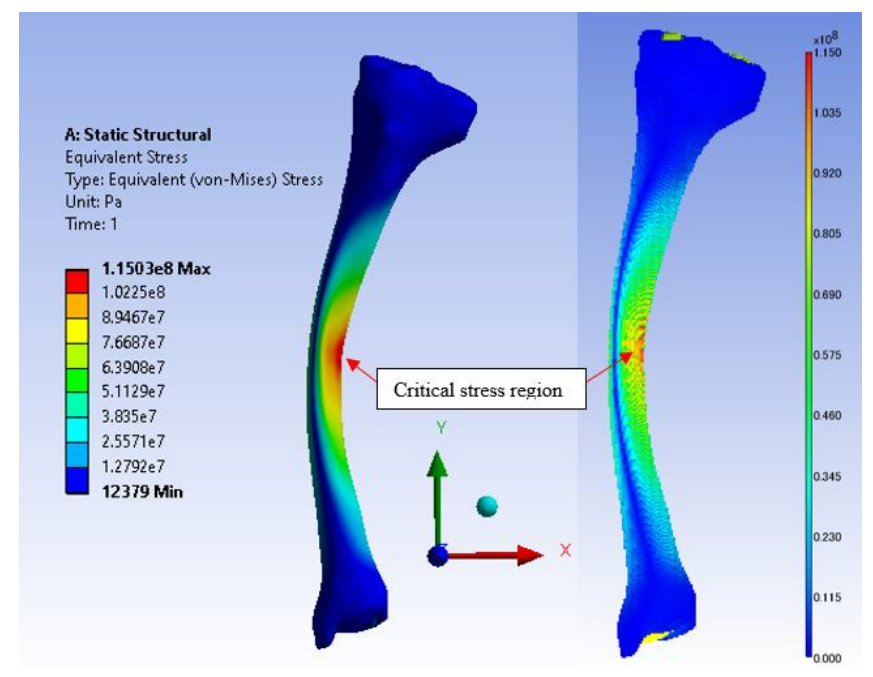

Figure 6: Stress distribution of polygon-based (left) and voxelbased (right) FE model during compression fracture load test

On top of that, it is observed that both of the FE models displayed identical contour band throughout the models. This indicates that both models predicted almost identical stress distribution when fracture due to compression takes place.

\subsection{Computing time demand during FEA}

Polygon-based and voxel-based OI tibia FE models require different amount of computing power. It was observed that both models had a huge difference in terms of time taken to complete one FEA run. For polygon-based models, the time taken during stance loading test were consistently recorded at below 15 seconds. Figure 7 shows the elapsed time for ANSYS Workbench to complete FEA for polygon-based model, the example taken was from $15^{\circ}$ stance loading test.

\begin{tabular}{|l|l|}
\hline Information \\
\hline Status & Done \\
\hline MAPDL Elapsed Time & $11 . \mathrm{s}$ \\
\hline
\end{tabular}

Figure 7: Time taken during stance loading test of polygonbased model $\left(15^{\circ}\right)$ 
However, for voxel-based model, the time taken for one complete FEA is much more than polygon-based models. It was observed that one complete FEA of voxel-based model would take between 4 to 6 minutes. Figure 8 shows the elapsed time for Voxelcon to complete FEA for voxel-based model, the example taken was from $15^{\circ}$ stance loading test as well.

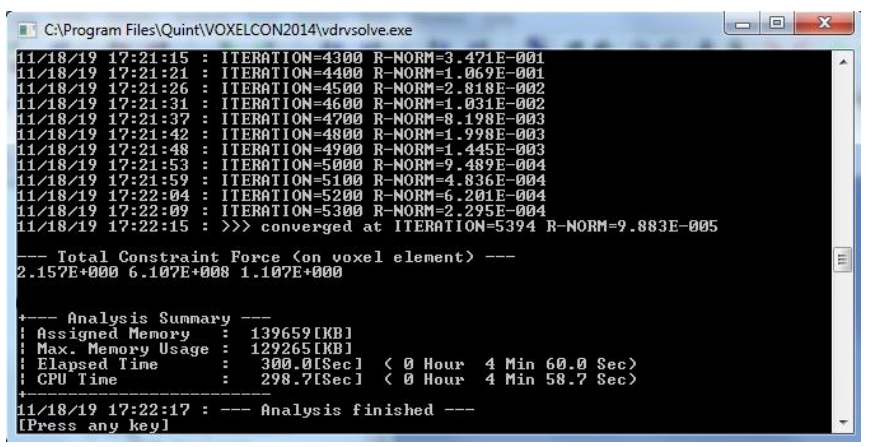

Figure 8: Time taken during stance loading test of voxel-based model $\left(15^{\circ}\right)$

Based on Figure 7 and 8, it was observed that for the same set of stance loading test, polygon-based model took $11 \mathrm{~s}$ for one complete FEA while voxel-based model took 300s. The time taken for voxel-based FEA was 45 times the amount of time needed for polygon-based FEA, even though the FEA tests were performed on identical models under similar boundary conditions. Also, it is worth mentioning that both types of FEA were performed using the same desktop computer.

Such situation was similar for other cases as well, where polygon-based FEA always take significantly less amount of time to complete compared to voxel-based FEA. For clinical usage such as surgical planning before rodding surgery for OI, the advantage of being able to conduct FEA within short amount of time. Doctors would be able to predict the timing of fracture of OI tibia after running multiple trials of FEA in a relatively short amount of time, compared to using voxelbased models. The lower computing demand of polygonbased FEA will also be beneficial by reducing the cost required to purchase high end computers for FEA purposes.

The comparison of FEA results have shown that both polygon-based and voxel-based FE models were capable to produce similar FE predictions. Most of the FEA results yielded percentage difference of $<1 \%$. While there were some outliers that are some results that are above $1 \%$, those are likely due to the difference between the area of load application and fixed support. Establishing identical boundary conditions for both types of FE models were a challenge due to the different methods in selecting surface for boundary condition. However, the resulting percentage difference were never more than $9 \%$, which is relatively low, seeing that FEA predictions are highly sensitive to the setup of boundary conditions [19], and slight variation of boundary condition setup could visibly affect the outcome of FEA.
This situation sets a path for future studies in developing strategies on refining the placement of boundary conditions.

\section{CONCLUSION}

The comparison between polygon-based and voxelbased FE models has proven that both models are able to produce similar prediction under identical boundary conditions. However, the computing time between two types of FEA differs by a huge margin, as in polygon-based FEA requires less time compared to voxel-based FEA, thus, less computing demand. With the capability to produce identical FEA predictions while keeping computing demands low, polygon-based FEA would be suitable for clinical usage such as surgery planning where multiple FEA trials are required. Therefore, strategies to convert patient-specific CT image of OI tibia into polygon-based FE model would be beneficial in practical usage due to its time saving aspect.

\section{ACKNOWLEDGMENT}

This work was financially supported by the Ministry of Education Malaysia under Fundamental Research Grant Scheme, FRGS/1/2016/TK03/UNIMAP/02/6.

\section{REFERENCES}

[1] D. O. Sillence, A. Senn, and D. M. Danks, "Genetic heterogeneity in osteogenesis imperfecta.," J. Med. Genet., vol. 16, no. 2, pp. 101-16, 1979.

https://doi.org/10.1136/jmg.16.2.101

[2] A. Alhousseini et al., "A Non-Lethal Osteogenesis Imperfecta Type II Mutation.," Gynecol. Obstet. Invest., vol. 48201, pp. 1-5, 2018.

[3] Rohit Bharat Patil and Girish Lonare, "Analysis of Four Wheeler Front Automotive Axle Through Finite Element Analysis," Int. J. Emerg. Trends Eng. Res., vol. 4, no. 4, pp. 18-21, 2016.

[4] Rohit Bharat Patil and Girish Lonare, "Static Analysis of Front Axle and Its Optimization Using Composite Materials," Int. J. Emerg. Trends Eng. Res., vol. 5, no. 10, pp. 26-29, 2017.

[5] E. Barua, S. Das, and A. B. Deoghare, "Development of computational Tibia model to investigate stress shielding effect at healing stages," Mater. Today Proc., vol. 5, no. 5, pp. 13267-13275, 2018.

https://doi.org/10.1016/j.matpr.2018.02.318

[6] F. Jm, V. Martinez, N. Graillon, L. Thollon, and L. Guyot, "Development and validation of an optimized finite element model of the human orbit," J. Stomatol. Oral Maxillofac. Surg., 2018.

[7] R. Hambli and S. Allaoui, "A robust 3D finite element simulation of human proximal femur progressive fracture under stance load with experimental validation," Ann. Biomed. Eng., vol. 41, no. 12, pp. 2515-2527, 2013.

https://doi.org/10.1007/s10439-013-0864-9 
[8] S. B. C. Wanna et al., "Prediction on fracture risk of femur with Osteogenesis Imperfecta using finite element models: Preliminary study," J. Phys. Conf. Ser., vol. 908, p. 012022, 2017.

[9] N. Mansor et al., "Reconstruction of Cortical and Cancellous Bone in Tibia with Osteogenesis Imperfecta," J. Telecommun. Electron. Comput. Eng., vol. 10, no. 1, pp. 115-119, 2018.

[10] H. S. Hosseini, D. H. Pahr, and P. K. Zysset, "Modeling and experimental validation of trabecular bone damage, softening and densification under large compressive strains," $J$. Mech. Behav. Biomed. Mater., vol. 15, pp. 93-102, 2012.

https://doi.org/10.1016/j.jmbbm.2012.06.005

[11] A. Fung, L. L. Loundagin, and W. B. Edwards, "Experimental validation of finite element predicted bone strain in the human metatarsal," $J$. Biomech., vol. 60, pp. 22-29, 2017.

[12] R. Su, G. M. Campbell, and S. K. Boyd, "Establishment of an architecture-specific experimental validation approach for finite element modeling of bone by rapid prototyping and high resolution computed tomography," Med. Eng. Phys., vol. 29, no. 4, pp. 480-490, 2007.

[13] S. Oliviero, M. Giorgi, and E. D. Ara, "Journal of the Mechanical Behavior of Biomedical Materials Validation of fi nite element models of the mouse tibia using digital volume correlation," J. Mech. Behav. Biomed. Mater., vol. 86, no. March, pp. 172184, 2018.

[14] S. B. C. Wanna, K. S. Basaruddin, M. H. M. Som, A. F. Salleh, and A. R. Sulaiman, "Effect of loading direction on fracture of bone with osteogenesis imperfecta (OI) during standing," AIP Conf. Proc., vol. 2030, no. June 2019, 2018. https://doi.org/10.1063/1.5066735

[15] S. B. C. Wanna et al., "Fracture risk prediction on children with Osteogenesis Imperfecta subjected to loads under activity of daily living," IOP Conf. Ser. Mater. Sci. Eng., vol. 429, no. 1, 2018.

[16] M. Lengsfeld, J. Schmitt, P. Alter, J. Kaminsky, and R. Leppek, "Comparison of geometry-based and CT voxel-based finite element modelling and experimental validation," Med. Eng. Phys., vol. 20, no. 7, pp. 515-522, 1998.

[17] L. H. Nguyen and D. Schillinger, "A multiscale predictor/corrector scheme for efficient elastoplastic voxel finite element analysis, with application to CT-based bone strength prediction," Comput. Methods Appl. Mech. Eng., vol. 330, pp. 598-628, 2018.

https://doi.org/10.1016/j.cma.2017.11.014

[18] I. Watanabe and A. Yamanaka, "Voxel coarsening approach on image-based finite element modeling of representative volume element," Int. J. Mech. Sci., vol. 150, no. October 2018, pp. 314-321, 2019.

[19] D. De Kegel, A. Meynen, N. Famaey, G. Harry van Lenthe, B. Depreitere, and J. Vander Sloten, "Skull fracture prediction through subject-specific finite element modelling is highly sensitive to model parameters," J. Mech. Behav. Biomed. Mater., vol. 100, no. August, p. 103384, 2019.

https://doi.org/10.1016/j.jmbbm.2019.103384

[20] I. Kutzner, B. Heinlein, F. Graichen, A. Bender, A. Rohlmann, and A. Halder, "Loading of the knee joint during activities of daily living measured in vivo in five subjects," J. Biomech., vol. 43, no. 11, pp. 2164-2173, 2010. https://doi.org/10.1016/j.jbiomech.2010.03.046

[21] J. M. Fritz, Y. Guan, M. Wang, P. A. Smith, and G. F. Harris, "A fracture risk assessment model of the femur in children with osteogenesis imperfecta (OI) during gait," Med. Eng. Phys., 2009. https://doi.org/10.1016/j.medengphy.2009.06.010 